Revista Arbitrada Interdisciplinaria KOINONIA

Año VII. Vol VII. N¹3. Enero - Junio. 2022

Hecho el depósito de Ley: FA2016000010 ISSN: 2542-3088

FUNDACIÓN KOINONIA (F.K). Santa Ana de Coro. Venezuela.

Yanedis Ruth Miranda-Núñez

http://dx.doi.org/10.35381/r.k.v7i13.1643

\title{
Aprendizaje significativo desde la praxis educativa constructivista
}

Meaningful learning from the constructivist educational praxis

\author{
Yanedis Ruth Miranda-Núñez \\ janedis7691@gmail.com \\ Institución Educativa San José No 2 Magangué-Bolívar \\ Colombia \\ https://orcid.org/0000-0002-5596-5729
}

Recibido: 15 de septiembre 2021

Revisado: 10 de noviembre 2021

Aprobado: 15 de diciembre 2021

Publicado: 01 de enero 2022 


\title{
RESUMEN
}

La presente investigación tuvo el propósito generar principios teóricos para una praxis educativa constructivista como generadora del aprendizaje significativo de las matemáticas en los estudiantes de Educación Básica Primaria. La metodología, enmarcada en el paradigma interpretativo y con enfoque cualitativo, se realizó mediante la aplicación del método fenomenológico. Los informantes clave fueron siete docentes del área de matemáticas de $5^{\circ}$ grado y catorce de sus estudiantes. Para recabar la información se utilizó la técnica de la entrevista en profundidad con base en un guion de preguntas. Las entrevistas se transcribieron, codificaron, categorizaron y triangularon para dar respuesta al propósito de investigación. Como resultado se obtuvo una conceptualización de los, principios teóricos para una praxis educativa constructivista generadora de aprendizaje significativo en el área de las matemáticas. Se considera que la reflexión del docente es el punto de partida para avanzar hacia una praxis educativa eficaz constructivista enseñanza de las matemáticas.

Descriptores: Educación; matemáticas; práctica pedagógica. (Tesauro UNESCO).

\begin{abstract}
The purpose of this research was to generate theoretical principles for a constructivist educational praxis as a generator of meaningful learning of mathematics in elementary school students. The methodology, framed in the interpretative paradigm and with a qualitative approach, was carried out through the application of the phenomenological method. The key informants were seven 5th grade mathematics teachers and fourteen of their students. The in-depth interview technique was used to collect the information based on a script of questions. The interviews were transcribed, codified, categorized and triangulated to respond to the research purpose. As a result, a conceptualization of the theoretical principles for a constructivist educational praxis that generates meaningful learning in the area of mathematics was obtained. It is considered that the teacher's reflection is the starting point to advance towards an effective constructivist educational praxis for the teaching of mathematics.
\end{abstract}

Descriptors: Education; mathematics; pedagogical practice. (UNESCO Thesaurus). 


\section{INTRODUCCIÓN}

El constructivismo, como marco filosófico y onto-epistemológico de la educación, implica una concepción del ser humano como constructor de su propio saber. Se trata de aceptar al estudiante como un sujeto protagonista de su aprendizaje, el cual se soporta en los esquemas o marcos conceptuales que trae y desde los cuales aprende. Dentro del constructivismo, tal como lo indican Ausubel, Novak y Hanesian (2000), el aprendizaje significativo consiste en un proceso mediante el cual el estudiante, para aprender, relaciona los conceptos nuevos con los que posee, así como los conceptos nuevos con la experiencia que tiene. Luego, el trabajo en el aula del docente consiste en organizar el espacio y los materiales y guiar al estudiante hacia el logro de su aprendizaje.

En este contexto, como refiere Miranda-Núñez (2020), enseñar y aprender matemáticas comprende la tríada estudiante-maestro-contenido como base para lograr la aproximación a los números y las formas necesarias para alcanzar el aprendizaje significativo que les permita a los estudiantes desarrollar las habilidades numéricas y aplicarlas en la vida cotidiana. Por otra parte, se requiere que el docente realice una praxis que guíe al estudiante en su proceso educativo, acrecentando sus fortalezas en la construcción del conocimiento.

A pesar de los esfuerzos gubernamentales y las reformas, no se ha logrado la meta que más del $25 \%$ de los estudiantes pasaran de niveles de desempeño no deseados (Insuficiente y Mínimo) a niveles deseados (Satisfactorio y Avanzado) en las áreas de lenguaje y matemáticas. Sumado a ello, se tiene que más del $50 \%$ de los estudiantes de la entidad Territorial de Magangué solo alcanzan el mínimo de las competencias de matemáticas y lenguaje. Además, se encuentran por debajo de los niveles nacionales, como lo indican el Instituto Colombiano para la evaluación de la educación (ICFES, 2017) y la Organización para la Cooperación y el Desarrollo Económicos (OECD, 2019).

Por otro lado, cabe destacar que, no existe una única teoría constructivista, por lo cual se puede definir el constructivismo como el conjunto de teorías psicológicas que conciben el conocimiento como una construcción activa del sujeto, resultado de su interacción con 
el ambiente, con los demás y consigo mismo. De este modo, el conocimiento es siempre una interacción entre la nueva información que se nos presenta y lo que ya sabíamos, y aprender es construir modelos para interpretar la información que recibimos. (Pozo, 1996, p. 60).

Así mismo, el constructivismo es un movimiento pedagógico que considera al aprendiz como un ente activo, capaz de construir su conocimiento sobre la base de sus potencialidades y experiencias, en conjunción con el contexto ambiental que lo rodea (Díaz y Hernández, 2003, p. 25). Por su parte, luego de haber analizado diversas definiciones de constructivismo, Coll y Martin (1997), lo definen como:

Un determinado enfoque o paradigma explicativo del psiquismo humano que subraya la importancia de la actividad mental constructiva de las personas en los procesos de adquisición de conocimientos y que, proyectado al ámbito de la educación, conduce a una visión del aprendizaje escolar como un proceso de construcción que el alumnado lleva a cabo a partir de sus conocimientos y experiencias previas y la enseñanza como ayuda a este proceso de construcción (p.15).

En este orden de ideas, según Díaz y Hernández (2010), el constructivismo es un movimiento pedagógico que abarca tres visiones distintas: constructivismo psicogenético (representado por Piaget) y referido a la equilibración de los procesos de asimilación y acomodación; el constructivismo cognitivo (Ausubel, Novak y Hanesian), relacionado con las habilidades del pensamiento y procesamiento de la información y, el constructivismo sociocultural (Vygotsky y también Bandura) que hace énfasis en cómo el individuo aprende por medio de la cooperación y su desenvolvimiento social. Cabe destacar que, si bien todas estas teorías y sus autores comparten la idea de que el ser humano es un ente activo durante el aprendizaje, ninguno de ellos se denominó como constructivista. Desde la perspectiva onto-epistemológica del constructivismo, en el contexto educativo, la concepción del docente y del estudiante como seres humanos constructores de conocimiento es determinante en la relación que se establece entre ellos y el contenido. Esta relación tiene lugar durante el proceso de enseñanza y aprendizaje en el aula, 
cuando el docente despliega su praxis educativa. De igual forma, la praxis educativa involucra las reflexiones del docente sobre su quehacer pedagógico, cómo lo hace, cuándo lo hace y por qué lo hace. Esto es así, sobre todo si se tiene en cuenta que su práctica está conformada por un cúmulo de acciones, conocimientos, sentimientos, creencias, valores que, consciente o inconscientemente, los cuales, como expone Miranda-Núñez (2020) manifiesta en el aula durante el proceso educativo, influyendo en el estudiante. Luego, reflexionar sobre su accionar es fundamental desde la visión constructivista, ya que siempre debe tener presente que su acción debe girar en torno a los intereses y necesidades del estudiante, más que centrarse en el contenido o en su propio saber. Por otro lado, Broitman, Escobar, Ponce y Sancha (2017), ofrecen un libro titulado Enseñar a estudiar matemáticas en la escuela primaria. Este libro, en tanto Cuaderno de apoyo didáctico, recoge sus experiencias y su visión constructivista de los procesos de enseñanza y aprendizaje de las matemáticas. Por eso, parten de una crítica sobre el método tradicional e indican que, para aprender matemáticas, el alumno debe trabajar conocimiento relacionado con las técnicas mecánicas e información. En consecuencia, desde tal postura, consideran al estudio de las matemáticas como un "eslabón" entre la enseñanza y el aprendizaje, donde la enseñanza no puede desentenderse de los procesos de aprendizaje de los alumnos. Es decir, no basta con "enseñar" (mostrar, informar, solicitar ejercitación), también se requiere un tiempo de trabajo y estudio por parte de los alumnos, tanto en el aula como fuera de ella. Este libro es de suma importancia por cuanto involucra el constructivismo, la praxis pedagógica del docente y el aprendizaje significativo de las matemáticas por los estudiantes. Las actividades propuestas ofrecen la oportunidad de comparar la praxis educativa constructivista del docente en su hacer; asimismo, indagar por las actividades de aprendizaje de las matemáticas que realizan los estudiantes, tanto en el aula como fuera de ella.

Otro aporte es el de López (2017) con su tesis doctoral titulada "Constructivismo como plataforma epistémica en didáctica alternativa en resolución de problemas matemáticos". 
El estudio, fundado en el constructivismo, está dirigido a estructurar una plataforma epistémica en didáctica alternativa para la resolución de problemas matemáticos, tomando como marco de intervención a la Educación Básica Venezolana. Su propósito general es generar una aproximación interpretativa sobre el constructivismo como plataforma epistémica en didáctica alternativa en la resolución de los problemas matemáticos, en la Educación Básica Venezolana.

Finalmente, merece traer a colación a Zamorano (2015), quien en su tesis doctoral titulada "La práctica de la enseñanza de las matemáticas a través de las situaciones de contingencia", tuvo como objetivo analizar situaciones de contingencia y la incidencia del conocimiento matemático del profesor para su gestión. Si bien el profesor planifica sus clases, durante el desarrollo de éstas ocurren situaciones no planificadas como las preguntas de los estudiantes a las que todo docente debe responder. Estas situaciones se denominan de contingencia. En ese sentido, los objetivos de esta investigación apuntan a una comprensión de la complejidad de la enseñanza para interpretar la gestión del profesor de matemáticas en situaciones de contingencia. Este estudio aporta a la presente investigación dos elementos; por una parte, expone la práctica de la enseñanza de la matemática en situaciones imprevistas como las preguntas de los estudiantes que exigen respuestas inmediatas. Por la otra, evidencia la importancia que tiene el conocimiento del contenido que se enseña, así como la forma de enseñarlo para el aprendizaje de los estudiantes. Es decir, permitió una visión de los procesos de enseñanza y aprendizaje de las matemáticas en el lugar donde ocurren: el salón de clases.

Se plantea como objetivo de la investigación generar principios teóricos para una praxis educativa constructivista como generadora del aprendizaje significativo de las matemáticas en los estudiantes de Educación Básica Primaria. 


\section{METODOLOGÍA}

La presente investigación se realizó dentro del paradigma, denominado por Yuni y Urbano (2005), fenomenológico, comprensivo, interpretativo. En él, desde la fenomenología se enfatiza el rol activo del sujeto cognoscente como constructor de conocimiento. La comprensión nos lleva a interpretar, dando significado a sus acciones, respetando sus ideas y palabras. En el marco del paradigma interpretativo de la investigación, ésta asume la investigación cualitativa como enfoque. En esta investigación, la dimensión metodológica procede mediante el método fenomenológico epistemológico. Como método, la fenomenología se opone al método natural que comienza por considerar al mundo como existente fuera de la conciencia y prescinde de ésta. Debido a que el método fenomenológico permite estudiar la realidad desde las vivencias y experiencias de los actores en su contexto y procede de manera inductiva, la investigación se encuentra inserta en la modalidad de investigación de campo. Los sujetos participantes, siendo ellos docentes y estudiantes de $5^{\circ}$ grado de básica primaria en Magangué, Bolívar. Los mismos se seleccionaron de forma intencional, en razón de su disponibilidad para participar en la investigación. Con relación a las personas como informantes, se seleccionó a 7 docentes de grado $5^{\circ}$, uno por cada institución, previa aceptación para participar en la investigación. Como técnica se realizó la entrevista en profundidad es la técnica privilegiada para obtener la información necesaria, y de hecho fue la técnica aplicada utilizando la aplicación de WhatsApp en los celulares.

\section{RESULTADOS}

Una praxis educativa de las matemáticas desde el constructivismo se soporta, según autores como Carretero (1997), Coll y Martin (1997), Porlán (1997), en la concepción de un estudiante que, al relacionar los conocimientos que posee con los nuevos contenidos matemáticos que le presentan la asignatura, debe ser capaz de construir un aprendizaje significativo de las matemáticas. El aprendizaje así logrado es transferible a otras áreas y a la vida cotidiana. (Ausubel, Novak y Hanesian, 2000). 
Los docentes aludieron la reflexión en su concepción; sin embargo, no está presente en los procesos de enseñanza y aprendizaje narrados. Luego, la praxis educativa que realizan no es, precisamente, una acción reflexiva, por lo que se aleja del concepto de praxis. Se infiere que aún existen docentes que realizan una praxis tradicional, evidenciada en actividades como una exposición magistral o la explicación docente.

\section{PROPUESTA}

Principios teóricos para una praxis educativa constructivista generadora de aprendizaje significativo en el área de las matemáticas. Los principios son entendidos como el punto de partida de un proceso de conocimiento. El proceso de conocimiento que se pretende fundamentar refiere a ¿cómo fundar una praxis educativa constructivista que genere un aprendizaje significativo en el área de las matemáticas? A modo de respuesta a la interrogante, se configuraron los principios de: Reflexividad, problematización, participación, significatividad, acompañamiento y evaluación auténtica. De manera sintetizada, los principios mencionados se pueden observar en el gráfico 1. En éste se destaca que todos convergen hacia el logro de una praxis educativa constructivista para lograr un aprendizaje significativo del estudiante. 


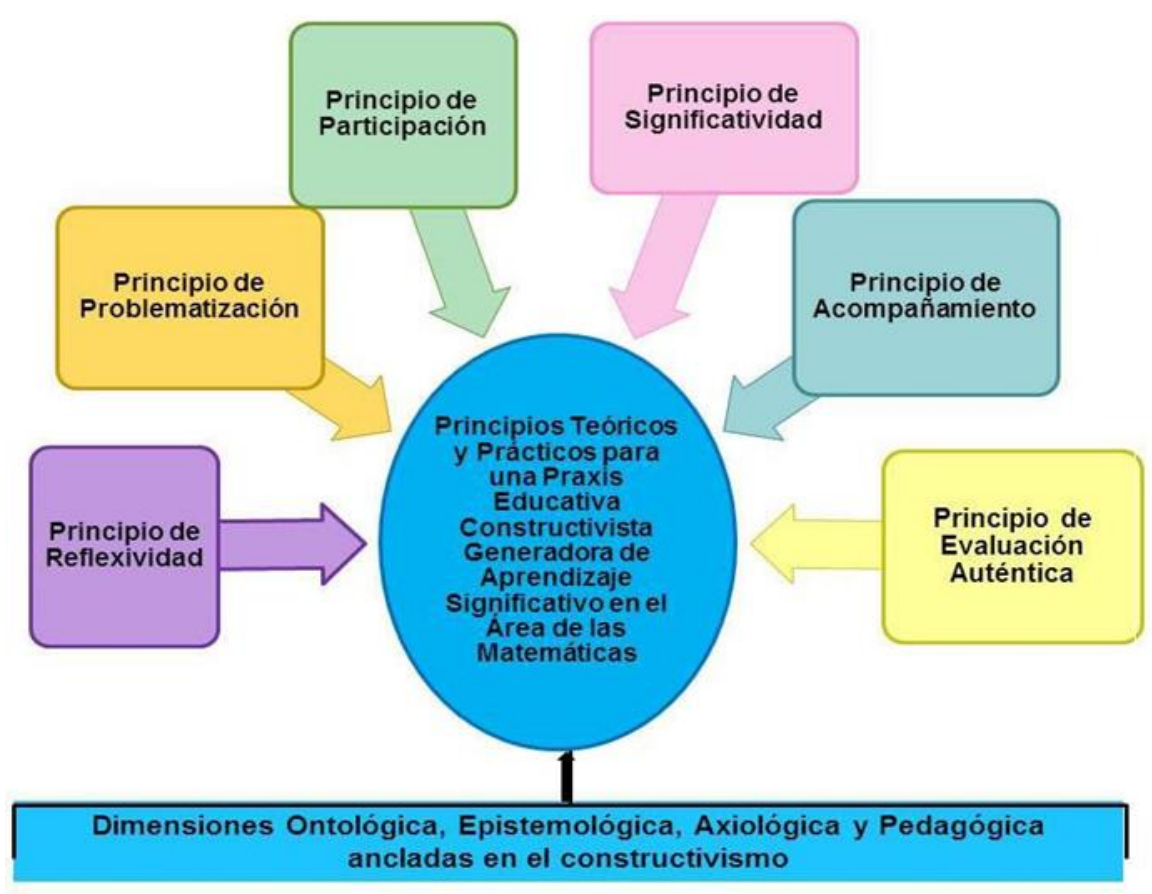

Figura 1. Principios para una praxis educativa constructivista generadora de aprendizaje significativo.

Elaboración: El autor.

Para la elaboración de los principios, la autora asumió plenamente las palabras de Martínez (2001), respecto que, en tanto formulación teórica, son una construcción mental simbólica, verbal o icónica, de naturaleza conjetural o hipotética, que nos obliga a pensar de un modo nuevo (p.242), puesto que para generarlos hizo gala de sus habilidades de pensamiento al establecer las relaciones pertinentes entre las categorías halladas durante la investigación y tomar en cuenta las dimensiones antes descritas. El desarrollo de cada uno de los principios ofrece una definición desde la perspectiva de una praxis educativa constructivista, un soporte teórico vinculado con el aprendizaje significativo de las matemáticas y, finalmente, el cómo llevarlo a la práctica, es decir, pensar en su aplicación por medio de actividades y estrategias apropiadas y contextualizadas. 


\section{CONCLUSIONES}

La investigación tuvo como centro de interés la praxis educativa constructivista para el logro de un aprendizaje significativo de las matemáticas. Desde la perspectiva del constructivismo se entiende al estudiante como una persona que ya posee ciertos conocimientos y quien, a partir de lo que sabe, es el constructor de su propio saber. Este modo de aprender y conocer implica, más que saber algo, el desarrollo de habilidades de pensamiento como analizar, sintetizar y, sobre todo, comparar y establecer relaciones. En consecuencia, el trabajo del docente es organizar el espacio y los materiales y acompañar al estudiante, guiándolo hacia el logro de un aprendizaje que pueda transferir a otras áreas del conocimiento y a su vida cotidiana.

Sin embargo, tanto la enseñanza constructivista como el aprendizaje significativo de las matemáticas siguen siendo un problema en el ámbito mundial. Existen evidencias, y los resultados de esta investigación es una de ellas, de que muchos docentes piensan que son constructivistas porque indagan por los conocimientos previos de los estudiantes, aunque luego dictan clase y los niños copian del tablero. Por otro lado, los estudiantes aprenden matemáticas de memoria, incluyendo algunos procedimientos, es decir, tal vez saben las operaciones básicas de matemáticas, pero no desarrollan las habilidades del pensamiento necesarias que les permitan utilizar esos conocimientos para resolver situaciones y problemas a lo largo de su vida.

Por eso, este estudio se llevó a cabo desde la investigación fenomenológica, con el fin de acceder a la experiencia vivida por los docentes y, desde ella, vislumbrar cómo la concepción o visión que poseen sobre su labor, guía su práctica y, consecuentemente, el trabajo escolar de los estudiantes. Las experiencias compartidas ayudan a repensar la práctica educativa como praxis y como praxis constructivista para cuestionarla, amarla y defenderla.

La praxis educativa constructivista se entiende como un hacer educativo propio e interactivo que involucra un docente mediador, estudiantes activos constructores de conocimiento y contenido matemático significativo, y, como acción reflexiva. Como 
manera de realizar el proceso pedagógico comprende un modo de actuar, una práctica centrada en el estudiante, un docente facilitador de aprendizajes y una práctica centrada en el contenido y las estrategias de enseñanza. Como acción reflexiva, involucra la reflexión, la reflexión sobre las estrategias de enseñanza y aprendizaje y la reflexión sobre su rol.

Al respecto, cabe destacar dos aspectos. Por una parte, el hecho de concebirla como una práctica centrada en el contenido y las estrategias de enseñanza, la aleja del constructivismo. Esta conclusión se corrobora en el uso que le dan los estudiantes al cuaderno de matemáticas: copiar del tablero lo que escriben los docentes, para copiar y realizar las tareas, individuales y grupales, dentro del aula o asignadas para la casa y tomar dictado.

Por la otra, comprenderla como acción reflexiva es coherente con la concepción del concepto de praxis. No obstante, las concepciones señalan una evolución, en algunos maestros más lenta que en otros, de una enseñanza tradicional centrada en el maestro hacia una enseñanza centrada en el estudiante y su aprendizaje. El rol del docente empieza a cambiar de ser un agente transmisor de conocimiento a otro facilitador, orientador del proceso educativo.

\section{FINANCIAMIENTO}

No monetario.

\section{AGRADECIMIENTO}

A la Universidad Pedagógica Experimental Libertador; por el apoyo en el desarrollo y fomento de la investigación. 


\section{REFERENCIAS CONSULTADAS}

Ausubel, D.; Novak, J. y Hanesian, H. (2000). Psicología educativa. Un punto de vista cognoscitivo [Educational psychology. A cognitive point of view]. México: Trillas.

Broitman, C., Escobar, M., Ponce, H., y Sancha, I. (2017). Enseñar a estudiar matemáticas en la escuela primaria. [Teaching mathematics in primary school]. Primera edición. Ciudad Autónoma de Buenos Aires: Santillana,

Coll, C. y Martin, J. (1997). Un Marco Referencial Psicológico para la Educación Escolar. La Concepción Constructivista del Aprendizaje y de la Enseñanza. [A Psychological Reference Framework for School Education. The Constructivist Conception of Learning and Teaching]. Madrid. Alianza.

Díaz, F. y Hernández, G. (2003). Estrategias Docentes para un Aprendizaje Significativo. Una Interpretación Constructivista. [Teaching Strategies for Meaningful Learning. A Constructivist Interpretation]. México. Mc. Graw-Hill

Díaz, F. y Hernández, G. (2010). Estrategias docentes para un aprendizaje significativo. [Teaching strategies for meaningful learning]. Tercera edición. México: McGrawHill Interamericana

Instituto Colombiano para la evaluación de la educación. (ICFES, 2017). Informe nacional resultados nacionales 2009, 2012-2017, Saber $3^{\circ}, 5^{\circ}$ y $9^{\circ} 2017$ [National report national results 2009, 2012-2017, Saber $3^{\circ}, 5^{\circ}$ and $\left.9^{\circ} 2017\right]$. Bogotá. Colombia

López, J. (2017). Constructivismo como plataforma epistémica en didáctica alternativa en resolución de problemas matemáticos [Constructivism as an epistemic platform in alternative didactics in solving mathematical problems]. Tesis Doctoral. Universidad de Carabobo. Venezuela.

Martínez, M. (2001). Comportamiento humano. Nuevos métodos de investigación [Human behavior. New research methods]. 2ª Edición. México: Editorial Trillas.

Miranda-Núñez, Y. (2020). Praxis educativa constructivista como generadora de Aprendizaje Significativo en el área de Matemática. [Constructivist educational praxis as a generator of Meaningful Learning in the area of Mathematics] CIENCIAMATRIA, Revista Interdisciplinaria de Humanidades, Educación, Ciencia y Tecnología, 6(1). Edición Especial. DOI 10.35381/cm.v6i1.299 
Organización para la Cooperación y el Desarrollo Económicos. (OECD, 2019). Programa para la Evaluación Internacional de Alumnos (PISA). [Programme for International Student Assessment (PISA)] Disponible en https://n9.cl/wt4wi

Pozo, J. (1996). Aprendices y maestros [Apprentices and masters]. Madrid: Alianza.

Yuni, J., Urbano, C. (2005). Investigación Etnográfica. Investigación-Acción. Mapas y Herramientas para conocer la Escuela [Ethnographic Research. Action. Maps and Tools to get to know the Schoo]. Córdoba: Brujas.

Zamorano. (2015) La práctica de la enseñanza de las matemáticas a través de las situaciones de contingencia [The practice of teaching mathematics through contingency situations]. Tesis doctoral. Universidad Autónoma de Barcelona 